\title{
PERCOLATION IN THE SIGNAL TO INTERFERENCE RATIO GRAPH
}

\author{
OLIVIER DOUSSE, * École Polytechnique Fédérale de Lausanne \\ MASSIMO FRANCESCHETTI,** University of California, San Diego \\ NICOLAS MACRIS, ${ }^{* * *}$ École Polytechnique Fédérale de Lausanne \\ RONALD MEESTER, ${ }^{* * * *}$ Vrije Universiteit Amsterdam \\ PATRICK THIRAN, ${ }^{* * * * *}$ École Polytechnique Fédérale de Lausanne
}

\begin{abstract}
Continuum percolation models in which pairs of points of a two-dimensional Poisson point process are connected if they are within some range of each other have been extensively studied. This paper considers a variation in which a connection between two points depends not only on their Euclidean distance, but also on the positions of all other points of the point process. This model has been recently proposed to model interference in radio communications networks. Our main result shows that, despite the infinite-range dependencies, percolation occurs in the model when the density $\lambda$ of the Poisson point process is greater than the critical density value $\lambda_{c}$ of the independent model, provided that interference from other nodes can be sufficiently reduced (without vanishing).
\end{abstract}

Keywords: Percolation; wireless network; interference; Poisson Boolean model

2000 Mathematics Subject Classification: Primary 82B43

Secondary 60K35; 90B 18

\section{Introduction}

Continuum percolation models originated with a paper of Gilbert [3], who considered the following construction of a random graph: each pair of points of a two-dimensional Poisson point process of density $\lambda$ is joined by an edge if the points are within a distance $2 r$ of each other. The motivation to introduce such a construction was to model networks of broadcasting stations that can exchange messages if they are within a certain range of each other. Gilbert proved a phase-transition behavior, namely the existence of a critical value $\lambda_{\mathrm{c}} \equiv \lambda_{\mathrm{c}}(r)$ for the density of the Poisson point process such that, for $\lambda>\lambda_{\mathrm{c}}$, an unbounded connected subgraph almost surely forms (i.e. the model percolates) and, so, the network can provide long-distance communication by multi-hopping messages along a path of connected stations. On the other hand, for $\lambda<\lambda_{\mathrm{c}}$ any connected component is bounded. Gilbert's results sparked a wide range

Received 19 October 2005; revision received 22 December 2005.

* Current address: Deutsche Telekom AG, Laboratories, Ernst-Reuter-Platz 7, D-10587 Berlin, Germany.

** Postal address: Electrical and Computer Engineering, University of California, San Diego, 9500 Gilman Drive, Mail Code 0407, La Jolla, CA 92093-0407, USA.

*** Postal address: School of Computer and Communication Sciences, Laboratoire de Théorie des Communications (LTHC), Station 14, EPFL, 1015 Lausanne, Switzerland.

**** Postal address: Department of Mathematics, Vrije Universiteit Amsterdam, De Boelelaan 1081a, 1081 HV Amsterdam, The Netherlands.

***** Postal address: School of Computer and Communication Sciences, Laboratory for Computer Communication and Applications (LCA), Station 14, EPFL, 1015 Lausanne, Switzerland. Email address: patrick.thiran@epfl.ch 
of interest and have been considerably extended by many mathematicians. We refer to [5] for a survey of the literature.

Gilbert's model applies to multi-hop wireless networks, when the circular discs centered at the Poisson points are considered as the radiation patterns of signals transmitted by the broadcasting stations. We pick two points of the Poisson process and label them as a transmitter $\boldsymbol{x}_{i}$ and a receiver $\boldsymbol{x}_{j}$. The transmitter $\boldsymbol{x}_{i}$ radiates a signal with intensity proportional to the power $P$ spent to generate the transmission. The signal diffuses isotropically in the environment and is then received by $\boldsymbol{x}_{j}$ with intensity $P$ multiplied by a loss factor $\ell\left(\boldsymbol{x}_{i}, \boldsymbol{x}_{j}\right) \leq 1$, due to isotropic dispersion and absorption in the environment. Furthermore, the reception mechanism is affected by noise, which means that $\boldsymbol{x}_{j}$ is able to detect the signal only if its intensity is sufficiently high compared to the environmental noise $N>0$. Assuming that $\ell\left(\boldsymbol{x}_{i}, \boldsymbol{x}_{j}\right)=\ell\left(\boldsymbol{x}_{j}, \boldsymbol{x}_{i}\right)$, we conclude that $\boldsymbol{x}_{i}$ and $\boldsymbol{x}_{j}$ are able to establish a communication link if the signal to noise ratio (SNR) is above a given threshold $T$, that is, if

$$
\mathrm{SNR}=\frac{P \ell\left(x_{i}, x_{j}\right)}{N}>T .
$$

It is reasonable to assume the loss factor $\ell(\boldsymbol{x}, \boldsymbol{y})$ to be a decreasing function of the Euclidean distance between $\boldsymbol{x}$ and $\boldsymbol{y}$. It follows that fixing the threshold $T$ is equivalent to fixing the radius $r$ of the discs in Gilbert's model.

From a practical viewpoint, however, this simple model does not account for interference effects that arise when all nodes transmit at the same time. In this case, all nodes can contribute to the amount of noise present at the receiver and increasing the density of the transmitters may not always be beneficial for connectivity. These observations motivated Dousse et al. [2] to introduce a dependent percolation model that can be described as follows.

Consider two points of a planar Poisson point process, $\boldsymbol{x}_{i}$ and $\boldsymbol{x}_{j}$, and assume that $\boldsymbol{x}_{i}$ wants to communicate a message to $\boldsymbol{x}_{j}$. At the same time, however, all other nodes $\boldsymbol{x}_{k}, k \neq i, j$, also transmit an interfering signal that reaches $\boldsymbol{x}_{j}$. We write the total interference term at $\boldsymbol{x}_{j}$ as $\gamma \sum_{k \neq i, j} P \ell\left(\boldsymbol{x}_{k}, \boldsymbol{x}_{j}\right)$, where $P$ is the transmitted power and $\gamma>0$ is a factor that depends on the technology adopted in the system. Node $\boldsymbol{x}_{j}$ can then successfully receive the message from $\boldsymbol{x}_{i}$ if the signal to noise plus interference ratio (SINR) is greater than a given threshold, that is, if

$$
\mathrm{SINR}=\frac{P \ell\left(\boldsymbol{x}_{i}, \boldsymbol{x}_{j}\right)}{N+\gamma \sum_{k \neq i, j} P \ell\left(\boldsymbol{x}_{k}, \boldsymbol{x}_{j}\right)}>T .
$$

A random graph is now constructed as follows. For each pair of Poisson points, the SINR level at both ends is computed and an undirected edge between the two is drawn if this exceeds the threshold $T$ in both cases. In this way, the presence of an edge indicates the possibility of direct bidirectional communication between the two nodes, while the presence of a path between two nodes in the graph indicates the possibility of multi-hop bidirectional communication. Note that the constructed random graph does not have the independence structure of Gilbert's model, because the presence of an edge between any pair of nodes now depends on the random positions of all other nodes in the plane that are causing interference, and not only on the two end-nodes of the link. Such dependencies, as we shall see, make the mathematical analysis of this kind of graph considerably more challenging. We call this model the SINR model.

It was shown by Dousse et al. [2] that, by taking $\lambda$ large enough, there exists a $\bar{\gamma}(\lambda)>0$ such that, for $\gamma<\bar{\gamma}$, the network percolates. In order to deal with the dependency structure of the model, however, Dousse et al. assumed that the function $\ell$ has bounded support. This allows the mathematical analysis to be considerably simplified and a standard coupling argument with 
a finite-range dependent percolation model to be used to immediately obtain the result. In fact, the main focus of [2] was not mathematical, but rather to present a novel model of engineering interest.

Nevertheless, we reasonably expect stronger results. If we denote by $\lambda_{\mathrm{c}}$ the critical density of the model when $\gamma=0$, then we expect percolation for all $\lambda>\lambda_{\mathrm{c}}$, by taking $\gamma>0$ sufficiently small. Furthermore, this should also be the case when the function $\ell$ has unbounded support.

Our contribution in this paper is to show that this is correct under the most general class of loss functions $\ell$. We remark that most of the difficulties that we need to overcome deal with the long-range dependencies introduced by the unbounded support of $\ell$. It shall be clear from the proof of Theorem 1 that a tight density threshold for percolation at $\lambda_{\mathrm{c}}$ is easy to obtain when bounded support is assumed. However, from physics we know that in reality attenuation of a signal does not have bounded support, which gives some applied motivation to our additional mathematical efforts.

\section{The main result}

In this paper, the underlying point process will always be a Poisson process with density $\lambda>0$. Further parameters of the model are $N, \gamma, P$, and $T$. In the sequel, we consider $N>0$, $P>0$, and $T>0$ fixed, and study the existence of a percolation phenomenon for varying values of $\lambda$ and $\gamma$ (the case where $N=0$ is briefly discussed in Section 3.4). The function $\ell$ is called the attenuation function and we assume it to satisfy the following conditions:

1. $\ell(\boldsymbol{x}, \boldsymbol{y})$ only depends on $|\boldsymbol{x}-\boldsymbol{y}|$, that is, $\ell(\boldsymbol{x}, \boldsymbol{y})=l(|\boldsymbol{x}-\boldsymbol{y}|)$ for some function $l: \mathbb{R}^{+} \rightarrow \mathbb{R}^{+}$,

2. $l(x) \leq 1$,

3. $l$ is continuous and, as long as it does not vanish, strictly decreasing.

These three properties characterize our physical model for wave propagation. In order to ensure that the model is not degenerate, we must impose, in addition, the following two conditions on $l$ :

4. $l(0)>T N / P$,

5. $\int_{0}^{\infty} x l(x) \mathrm{d} x<\infty$.

Some comments on these conditions are perhaps necessary. If Condition 4 is not satisfied, then (1) never holds. Furthermore, the sum in the denominator of (1) is almost surely finite if and only if Condition 5 is satisfied (see, e.g. [1]).

We remark at this point that the lengths of the edges are uniformly bounded: when $\ell(\boldsymbol{x}, \boldsymbol{y}) \leq$ $T N / P$, no edge can form between $\boldsymbol{x}$ and $\boldsymbol{y}$. In [2] it was also shown that the degree of any vertex is bounded above uniformly by $1+1 / T \gamma$; the proof there is also valid in the case of unbounded support of $\ell$.

We write $\boldsymbol{x} \longleftrightarrow \boldsymbol{y}$ if there exists a sequence $\boldsymbol{x}_{1}, \boldsymbol{x}_{2}, \ldots, \boldsymbol{x}_{k}$ of Poisson points such that $\boldsymbol{x}_{1}=\boldsymbol{x}, \boldsymbol{x}_{k}=\boldsymbol{y}$, and $\boldsymbol{x}_{l}$ is connected by an edge to $\boldsymbol{x}_{l+1}$ for $1 \leq l<k$. A (connected) component or cluster is a set $\left\{\boldsymbol{x}_{i}: i \in J\right\}$ of points which is maximal with the property that $\boldsymbol{x}_{i} \longleftrightarrow \boldsymbol{x}_{j}$ for all $i, j \in J$.

As mentioned before, we denote by $\lambda_{\mathrm{c}}$ the critical density of the model when $\gamma=0$ (and all other parameters, $N, P$, and $T$, are fixed). It is known that for $\lambda \leq \lambda_{\mathrm{c}}$ we have no infinite cluster almost surely, while for $\lambda>\lambda_{\mathrm{c}}$ there is an infinite cluster with probability 1 . If there is an 
unbounded component of points with positive probability, we say that the signal to interference ratio graph percolates. (In fact, standard results from ergodic theory say that the existence of an unbounded component with positive probability implies that this latter probability is equal to 1 . For us, this is not immediately relevant.) We now present our main result.

Theorem 1. Let $\lambda_{\mathrm{c}}$ be the critical node density when $\gamma=0$ and assume that the attenuation function $\ell$ satisfies Conditions $1-5$ above. Then, for any node density $\lambda>\lambda_{\mathrm{c}}$, there exists a $\gamma^{*}(\lambda)>0$ such that, for $\gamma \leq \gamma^{*}(\lambda)$, the SINR model percolates.

\section{Proof of Theorem 1}

The main strategy of the proof is coupling the model to a discrete edge percolation model on the grid. By doing so, we end up with a dependent discrete model such that the existence of an infinite connected component in the edge percolation model implies the existence of an infinite connected component in the original graph. Although the edges of the discrete model are not finite-range dependent, we show that the probability of having a collection of $n$ closed edges in the discrete model decreases exponentially as $q^{n}$, where $q$ can be made arbitrarily small by appropriate choice of the parameters, and, therefore, that the existence of an infinite connected component follows from a Peierls argument.

We describe the construction of the discrete model first, then prove the percolation of this model, and lastly show the final result by coupling it with the SINR model.

\subsection{Mapping on a lattice}

If we set $\gamma=0$, then we obtain a fixed-radius Poisson Boolean model with radius $r_{\mathrm{B}}$ given by

$$
2 r_{\mathrm{B}}=l^{-1}\left(\frac{T N}{P}\right) .
$$

Since $l$ is continuous, strictly monotone, and larger than $T N / P$ at the origin, it follows that $l^{-1}(T N / P)$ exists.

We consider next a supercritical Boolean model $\mathcal{B}\left(\lambda, r_{\mathrm{B}}\right)$ with radius $r_{\mathrm{B}}$, where the node density $\lambda$ is higher than the critical value $\lambda_{\mathrm{c}}$. By rescaling the model, we can establish that the critical radius for a fixed density $\lambda$ is

$$
r^{*}(\lambda)=\sqrt{\frac{\lambda_{\mathrm{c}}}{\lambda}} r_{\mathrm{B}}<r_{\mathrm{B}} .
$$

Therefore, a Boolean model $\mathscr{B}(\lambda, r)$ with density $\lambda$ and radius $r$ satisfying $r^{*}(\lambda)<r<r_{\mathrm{B}}$ is still supercritical.

We map the latter model onto a discrete percolation model as follows. For $d>0$, we denote by $\mathcal{L}_{d}$ the two-dimensional square lattice whose vertices are located at all points of the form $(d x, d y)$ with $(x, y) \in \mathbb{Z}^{2}$. For each horizontal edge $a$ of $\mathcal{L}_{d}$, we denote by $z_{a}=\left(x_{a}, y_{a}\right)$ the point in the middle of the edge, and introduce a random field $A_{a}$, indexed by the edges of $\mathcal{L}_{d}$, that takes the value 1 if the following two events (illustrated in Figure 1) occur, and takes the value 0 otherwise:

1. the rectangle $\left[x_{a}-3 d / 4, x_{a}+3 d / 4\right] \times\left[y_{a}-d / 4, y_{a}+d / 4\right]$ is crossed from left to right by an occupied component in $\mathcal{B}(\lambda, r)$, 


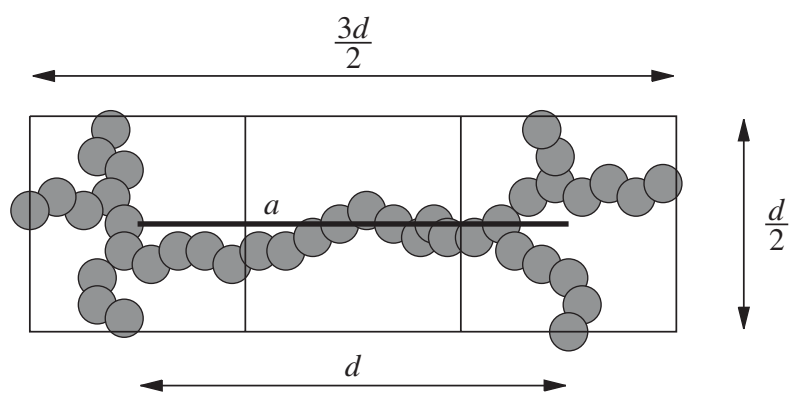

Figure 1: A horizontal edge $a$ that fulfils the two conditions for having $A_{a}=1$.

2. both squares $\left[x_{a}-3 d / 4, x_{a}-d / 4\right] \times\left[y_{a}-d / 4, y_{a}+d / 4\right]$ and $\left[x_{a}+d / 4, x_{a}+3 d / 4\right] \times$ $\left[y_{a}-d / 4, y_{a}+d / 4\right]$ are crossed from top to bottom by an occupied component in $\mathcal{B}(\lambda, r)$.

We define $A_{a}$ similarly for vertical edges, by swapping the horizontal and vertical coordinates in the above conditions.

According to [5, Corollary 4.1], the probability that $A_{a}=1$ can be made as large as we like by choosing $d$ large. The variables $A_{a}$ are not independent in general. However, if $a$ and $b$ are not adjacent, then $A_{a}$ and $A_{b}$ are independent; these variables thus define a 1-dependent edge percolation process.

We next define a second random field, $B_{a}$, again indexed by the edges in $\mathcal{L}_{d}$. We first define $\tilde{l}$, a shifted version of $l$, as follows:

$$
\tilde{l}(x)= \begin{cases}l(0), & x \leq \frac{\sqrt{10} d}{4}, \\ l\left(x-\frac{\sqrt{10} d}{4}\right), & x>\frac{\sqrt{10} d}{4} .\end{cases}
$$

We define the shot noise processes $I$ and $\tilde{I}$ as follows:

$$
\begin{aligned}
& I(z)=\sum_{k} l\left(\left|z-\boldsymbol{x}_{k}\right|\right), \\
& \tilde{I}(z)=\sum_{k} \tilde{l}\left(\left|z-\boldsymbol{x}_{k}\right|\right),
\end{aligned}
$$

where $z \in \mathbb{R}^{2}$ is an arbitrary point and the sum is over all points of the Poisson process $X$. Note that the shot noises are random variables, since they depend on the random positions of the points of $X$.

We now define the second random field $B_{a}$ as taking the value 1 if the value of the shot noise $\tilde{I}\left(z_{a}\right)$ does not exceed a certain threshold $M$, and taking the value 0 otherwise. As the distance between any point $z$ inside the rectangle $R\left(z_{a}\right)=\left[x_{a}-3 d / 4, x_{a}+3 d / 4\right] \times\left[y_{a}-d / 4, y_{a}+d / 4\right]$ and its center $z_{a}$ is at most $\sqrt{10} d / 4$, the triangle inequality implies that $\left|z_{a}-\boldsymbol{x}_{k}\right| \leq \sqrt{10} d / 4+$ $\left|z-\boldsymbol{x}_{k}\right|$ and, thus, that $I(z) \leq \tilde{I}\left(z_{a}\right)$ for all $z \in R\left(z_{a}\right)$. Therefore, $B_{a}=1$ implies that $I(z) \leq M$ for all $z \in R\left(z_{a}\right)$. Later, we will make an appropriate choice firstly for $d$ and then for $M$. 


\subsection{Percolation in the lattice}

For any edge $a$ of $\mathcal{L}_{d}$, we call the edge open if $C_{a}=A_{a} B_{a}=1$, that is, if both of the following events occur: there exist crossings in the rectangle $R\left(z_{a}\right)$ as described above and the shot noise is bounded by $M$ for all points inside $R\left(z_{a}\right)$. An edge $a$ that is not open is closed. We want to show that, for an appropriate choice of the parameters $M$ and $d$, there exists an infinite connected component of open edges at the origin, with positive probability.

To do this, we need an exponential bound on the probability of a collection of $n$ closed edges. Most of the difficulty of obtaining this resides in the infinite-range dependencies introduced by the random variables $B_{i}$. A careful application of Campbell's theorem will take care of this.

Consider any collection of $n$ edges $a_{1}, \ldots, a_{n}$. To keep the notation simple, we write $A_{a_{i}}=A_{i}, B_{a_{i}}=B_{i}$, and $C_{a_{i}}=C_{i}, i=1, \ldots, n$. In Proposition 3, below, we will prove that the probability that all these edges are closed simultaneously decreases exponentially with $n$. To do this, we first prove this for the fields $A$ and $B$.

Proposition 1. There exists a constant, $q_{A}<1$, such that, for any collection $\left\{a_{i}\right\}_{i=1}^{n}$ of $n$ distinct edges, we have

$$
\mathrm{P}\left(A_{1}=0, A_{2}=0, \ldots, A_{n}=0\right) \leq q_{A}^{n},
$$

where $\left\{A_{i}\right\}_{i=1}^{n}$ denotes the random variables of the field A associated with them. Furthermore, for any $\varepsilon>0$, we can choose d large enough that $q_{A} \leq \varepsilon$.

Proof. This proposition follows directly from the observation that it is always possible to find a subset of indices $\left\{k_{j}\right\}_{j=1}^{m}$, with $1 \leq k_{j} \leq n$ for each $j$, such that the variables $\left\{A_{k_{j}}\right\}_{j=1}^{m}$ are independent and such that $m \geq n / 4$. Therefore, we have

$$
\begin{aligned}
\mathrm{P}\left(A_{1}=0, A_{2}=0, \ldots, A_{n}=0\right) & \leq \mathrm{P}\left(A_{k_{1}}=0, A_{k_{2}}=0, \ldots, A_{k_{m}}=0\right) \\
& =\mathrm{P}\left(A_{1}=0\right)^{m} \\
& \leq \mathrm{P}\left(A_{1}=0\right)^{n / 4} \\
& =: q_{A}^{n} .
\end{aligned}
$$

Furthermore, since $q_{A}=\mathrm{P}\left(A_{1}=0\right)^{1 / 4}$, it follows from [5, Corollary 4.1] that $q_{A}$ tends to zero as $d$ tends to infinity.

Proposition 2. There exists a constant, $q_{B}<1$, such that, for any collection $\left\{a_{i}\right\}_{i=1}^{n}$ of $n$ distinct edges, we have

$$
\mathrm{P}\left(B_{1}=0, B_{2}=0, \ldots, B_{n}=0\right) \leq q_{B}^{n},
$$

where $\left\{B_{i}\right\}_{i=1}^{n}$ denotes the random variables of the field $B$ associated with them. Furthermore, for any $\varepsilon>0$ and fixed $d$, we can choose $M$ large enough that $q_{B} \leq \varepsilon$.

Proof. To simplify the notation, we denote by $z_{i}$ the center, $z_{a_{i}}$, of the edge $a_{i}$. By Markov's inequality we have, for any $s \geq 0$,

$$
\begin{aligned}
\mathrm{P}\left(B_{1}=0, B_{2}=0, \ldots, B_{n}=0\right) & =\mathrm{P}\left(\tilde{I}\left(z_{1}\right)>M, \tilde{I}\left(z_{2}\right)>M, \ldots, \tilde{I}\left(z_{n}\right)>M\right) \\
& \leq \mathrm{P}\left(\sum_{i=1}^{n} \tilde{I}\left(z_{i}\right)>n M\right) \\
& \leq \exp (-s n M) \mathrm{E}\left(\exp \left(s \sum_{i=1}^{n} \tilde{I}\left(z_{i}\right)\right)\right)
\end{aligned}
$$


Applying Campbell's theorem (see, e.g. [6, p. 28]) to the function

$$
f(\boldsymbol{x})=\sum_{i=1}^{n} \tilde{l}\left(\left|z_{i}-\boldsymbol{x}\right|\right)
$$

we obtain

$$
\mathrm{E}\left(\exp \left(s \sum_{i=1}^{n} \tilde{I}\left(\boldsymbol{z}_{i}\right)\right)\right)=\exp \left(\lambda \int_{\mathbb{R}^{2}}\left(\exp \left(s \sum_{i=1}^{n} \tilde{l}\left(\left|\boldsymbol{x}-\boldsymbol{z}_{i}\right|\right)\right)-1\right) \mathrm{d} \boldsymbol{x}\right) .
$$

We need to estimate the exponent $s \sum_{i=1}^{n} \tilde{l}\left(\left|\boldsymbol{x}-\boldsymbol{z}_{i}\right|\right)$. As the $\left\{z_{i}\right\}$ are centers of edges, they are located on a square lattice with edge length $d / \sqrt{2}$. So, if we consider the square in which $\boldsymbol{x}$ is located, the contribution to $\sum_{i=1}^{n} \tilde{l}\left(\left|\boldsymbol{x}-\boldsymbol{z}_{i}\right|\right)$ coming from the four corners of this square is, at most, equal to 4 , since $\tilde{l}(x) \leq 1$. Around this square, there are 12 nodes, each located at a distance of at least $d / \sqrt{2}$ from $\boldsymbol{x}$. Further away, there are 20 other nodes at distances of at least $2 d / \sqrt{2}$ from $\boldsymbol{x}$, and so on. Consequently,

$$
\begin{aligned}
\sum_{i=1}^{n} \tilde{l}\left(\left|\boldsymbol{x}-\boldsymbol{z}_{i}\right|\right) & \leq \sum_{i=1}^{\infty} \tilde{l}\left(\left|\boldsymbol{x}-\boldsymbol{z}_{i}\right|\right) \\
& \leq 4+\sum_{k=1}^{\infty}(4+8 k) \tilde{l}\left(\frac{k d}{\sqrt{2}}\right) \\
& =: K .
\end{aligned}
$$

Now Condition 5 , above, on $l$ can easily be extended to $\tilde{l}$, and we clearly have

$$
\int_{y}^{\infty} x \tilde{l}(x) \mathrm{d} x<\infty, \quad \text { for some } y>0 .
$$

Using the integral criterion and (3), we conclude that the sum converges and, thus, $K<\infty$.

The computation above holds for any $s \geq 0$. We now take $s=1 / K$, so that

$$
s \sum_{i=1}^{n} \tilde{l}\left(\left|x-z_{i}\right|\right) \leq 1,
$$

for all $\boldsymbol{x}$. Furthermore, since $\mathrm{e}^{x}-1<2 x$ for all $x \leq 1$, we have

$$
\exp \left(s \sum_{i=1}^{n} \tilde{l}\left(\left|\boldsymbol{x}-\boldsymbol{z}_{i}\right|\right)\right)-1<2 s \sum_{i=1}^{n} \tilde{l}\left(\left|\boldsymbol{x}-\boldsymbol{z}_{i}\right|\right)=\frac{2}{K} \sum_{i=1}^{n} \tilde{l}\left(\left|\boldsymbol{x}-\boldsymbol{z}_{i}\right|\right) .
$$

Substituting this into (2), we obtain

$$
\begin{aligned}
\mathrm{E}\left(\exp \left(\sum_{i=1}^{n} \frac{\tilde{I}\left(z_{i}\right)}{K}\right)\right) & \leq \exp \left(\lambda \int_{\mathbb{R}^{2}} \frac{2}{K} \sum_{i=1}^{n} \tilde{l}\left(\left|\boldsymbol{x}-\boldsymbol{z}_{i}\right|\right) \mathrm{d} \boldsymbol{x}\right) \\
& =\exp \left(\frac{2 n \lambda}{K} \int_{\mathbb{R}^{2}} \tilde{l}(|\boldsymbol{x}|) \mathrm{d} \boldsymbol{x}\right) \\
& =\left[\exp \left(\frac{2 \lambda}{K} \int_{\mathbb{R}^{2}} \tilde{l}(|\boldsymbol{x}|) \mathrm{d} \boldsymbol{x}\right)\right]^{n} .
\end{aligned}
$$


Putting things together, we obtain

$$
\begin{aligned}
& \mathrm{P}\left(\tilde{I}\left(z_{1}\right)>M, \tilde{I}\left(z_{2}\right)>M, \ldots, \tilde{I}\left(z_{n}\right)>M\right) \\
& \quad \leq \exp (-s n M) \mathrm{E}\left(\exp \left(s \sum_{i=1}^{n} \tilde{I}\left(z_{i}\right)\right)\right) \\
& \quad \leq \exp \left(-\frac{n M}{K}\right)\left[\exp \left(\frac{2 \lambda}{K} \int_{\mathbb{R}^{2}} \tilde{l}(|\boldsymbol{x}|) \mathrm{d} \boldsymbol{x}\right)\right]^{n} \\
& \quad=q_{B}^{n},
\end{aligned}
$$

where $q_{B}$ is defined as

$$
q_{B}:=\exp \left(\frac{2 \lambda}{K} \int \tilde{l}(|\boldsymbol{x}|) \mathrm{d} \boldsymbol{x}-\frac{M}{K}\right) .
$$

Finally, it is easy to observe that this expression tends to zero as $M$ tends to infinity (for fixed $d$ and, hence, since $K$ depends only on $d$, for fixed $K$ ).

We next combine Propositions 1 and 2, in order to obtain a similar result for the field $C$.

Proposition 3. There exists a constant, $q_{C}<1$, such that, for any collection $\left\{a_{i}\right\}_{i=1}^{n}$ of $n$ distinct edges, we have

$$
\mathrm{P}\left(C_{1}=0, C_{2}=0, \ldots, C_{n}=0\right) \leq q_{C}^{n},
$$

where $\left\{C_{i}\right\}_{i=1}^{n}$ denotes the random variables of the field $C$ associated with them. Furthermore, for any $\varepsilon>0$, we can choose $d$ and $M$ so that $q_{C} \leq \varepsilon$.

Proof. For convenience, in the following calculations we introduce the notation $\bar{A}_{i}=1-A_{i}$ and $\bar{B}_{i}=1-B_{i}$. Firstly observe that

$$
1-C_{i}=1-A_{i} B_{i} \leq\left(1-A_{i}\right)+\left(1-B_{i}\right)=\bar{A}_{i}+\bar{B}_{i} .
$$

Let us denote by $p(n)$ the probability that we want to bound, and let $\left(k_{i}\right)_{i=1}^{n}$ be a binary sequence (i.e. $k_{i}=0$ or $k_{i}=1$ ) of length $n$. We denote by $\mathcal{K}$ the set of the $2^{n}$ such sequences. Then we can write

$$
\begin{aligned}
p(n) & =\mathrm{P}\left(C_{1}=0, C_{2}=0, \ldots, C_{n}=0\right) \\
& =\mathrm{E}\left(\left(1-C_{1}\right)\left(1-C_{2}\right) \cdots\left(1-C_{n}\right)\right) \\
& \leq \mathrm{E}\left(\left(\bar{A}_{1}+\bar{B}_{1}\right)\left(\bar{A}_{2}+\bar{B}_{2}\right) \cdots\left(\bar{A}_{n}+\bar{B}_{n}\right)\right) \\
& =\sum_{\left(k_{i}\right) \in \mathcal{K}} \mathrm{E}\left(\prod_{\left\{i: k_{i}=0\right\}} \bar{A}_{i} \prod_{\left\{i: k_{i}=1\right\}} \bar{B}_{i}\right) \\
& \leq \sum_{\left(k_{i}\right) \in \mathcal{K}} \sqrt{\mathrm{E}\left(\prod_{\left\{i: k_{i}=0\right\}} \bar{A}_{i}^{2}\right) \mathrm{E}\left(\prod_{\left\{i: k_{i}=1\right\}} \bar{B}_{i}^{2}\right)} \\
& =\sum_{\left(k_{i}\right) \in \mathcal{K}} \sqrt{\mathrm{E}\left(\prod_{\left\{i: k_{i}=0\right\}} \bar{A}_{i}\right) \mathrm{E}\left(\prod_{\left\{i: k_{i}=1\right\}} \bar{B}_{i}\right),}
\end{aligned}
$$


where the two last inequalities follow, respectively, from Schwartz's inequality and from the observation that $\bar{A}_{i}^{2}=\bar{A}_{i}$ and $\bar{B}_{i}^{2}=\bar{B}_{i}$. Applying Propositions 1 and 2 , we can bound each expectation in the sum. We thus obtain

$$
\begin{aligned}
p(n) & \leq \sum_{\left(k_{i}\right) \in \mathcal{K}} \sqrt{\prod_{\left\{i: k_{i}=0\right\}} q_{A} \prod_{\left\{i: k_{i}=1\right\}} q_{B}} \\
& =\sum_{\left(k_{i}\right) \in \mathcal{K}} \prod_{\left\{i: k_{i}=0\right\}} \sqrt{q_{A}} \prod_{\left\{i: k_{i}=1\right\}} \sqrt{q_{B}} \\
& =\left(\sqrt{q_{A}}+\sqrt{q_{B}}\right)^{n} \\
& =: q_{C}^{n}
\end{aligned}
$$

Firstly choosing $d$ large, and then $M$ appropriately, we can make $q_{C}$ smaller than any given $\varepsilon$.

With Proposition 3, the existence of percolation in our dependent bond percolation model follows from standard arguments. Indeed, with our exponential bound in Proposition 3, we can apply the usual Peierls argument (see, e.g. [4, pp. 16-19]) to establish the existence of percolation for appropriate $M$ and $d$.

\subsection{Percolation of the SINR model}

To conclude the proof of Theorem 1, we need to show that percolation of $C_{a}$ implies percolation in the SINR model, with appropriate $\gamma$. If $B_{a}=1$ then the interference level in the rectangle $R\left(z_{a}\right)$ is, at most, equal to $M$. Therefore, for two nodes $\boldsymbol{x}_{i}$ and $\boldsymbol{x}_{j}$ in $R\left(z_{a}\right)$ such that $\left|\boldsymbol{x}_{i}-\boldsymbol{x}_{j}\right| \leq 2 r$, we have

$$
\begin{aligned}
\frac{P l\left(\left|\boldsymbol{x}_{i}-\boldsymbol{x}_{j}\right|\right)}{N+\gamma \sum_{k \neq i, j} P l\left(\left|\boldsymbol{x}_{k}-\boldsymbol{x}_{j}\right|\right)} & \geq \frac{P l\left(\left|\boldsymbol{x}_{i}-\boldsymbol{x}_{j}\right|\right)}{N+\gamma P M} \\
& \geq \frac{P l(2 r)}{N+\gamma P M} .
\end{aligned}
$$

As $r<r_{\mathrm{B}}$ and as $l$ is strictly decreasing, we choose

$$
\gamma=\frac{N}{P M}\left(\frac{l(2 r)}{l\left(2 r_{\mathrm{B}}\right)}-1\right)>0,
$$

yielding

$$
\frac{P l(2 r)}{N+\gamma P M}=\frac{P l\left(2 r_{\mathrm{B}}\right)}{N}=T .
$$

Therefore, there exists a positive value of $\gamma$ such that any two nodes separated by a distance less than $r$ are connected in the SINR model. This means that in the rectangle $R\left(z_{a}\right)$ all connections of $\mathcal{B}(\lambda, r)$ also exist in the SINR model.

Finally, if $A_{a}=1$, there exist crossings along edge $a$, as shown in Figure 1. These crossings are designed such that if, for two adjacent edges $a$ and $b, A_{a}=1$ and $A_{b}=1$, then the crossings overlap, and they all belong to the same connected component (see Figure 2). Thus, an infinite cluster of such edges implies an infinite cluster in the Boolean model of radius $r$ and density $\lambda$. Since all edges $a$ of the infinite cluster of the discrete model are such that $A_{a}=1$ and $B_{a}=1$, this means that the crossings also exist in the SINR model, and thus form an infinite connected component. 


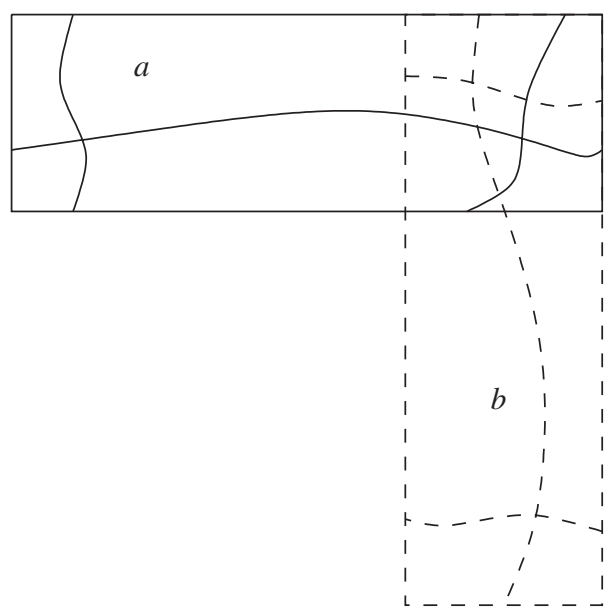

Figure 2: Two adjacent edges $a$ (solid) and $b$ (dashed) with $A_{a}=1$ and $A_{b}=1$. The crossings overlap, and form a connected component.

\subsection{The case where $N=0$}

It is clear that the model degenerates to trivial particular cases if we set $P=0$ or $T=0$. However, it is interesting to look at the case where there is no background noise, i.e. $N=0$, as the length of the edges is unbounded in this case. The proof given in the above sections can easily be adapted to this case. The only difference is that there is no Boolean threshold $\lambda_{\mathrm{c}}$ and, thus, the result holds for any density $\lambda>0$.

Corollary 1. Let $N=0$, and assume that the attenuation function $\ell$ satisfies Conditions $1-5$ as in Theorem 1. Then, for any node density $\lambda>0$, there exists $a \gamma^{*}(\lambda)>0$ such that, for $\gamma \leq \gamma^{*}(\lambda)$, the SINR model percolates.

\section{Conclusion and possible extensions}

In this paper, we proved that a percolation phenomenon occurs for some values of the parameters $\lambda$ (node density) and $\gamma$ (weight of the interference term) in the SINR model. When $\gamma=0$, the model reduces to a standard Boolean model, and it is known that there exists a $\lambda_{\mathrm{c}}$ such that percolation occurs whenever $\lambda>\lambda_{\mathrm{c}}$. We showed that, for any density $\lambda>\lambda_{\mathrm{c}}$, we can pick $\gamma$ small enough (but nonzero) that percolation still occurs.

We have thus improved the results of [2] in two ways: firstly we extended the range of node densities where a percolation phenomenon is proved to exist to the actual range where percolation can occur, and secondly we established the result for a large class of attenuation functions (in particular with unbounded support) that includes all isotropic, continuous, and strictly decreasing functions bounded from above by 1 .

We now present a summary of what is known about the set of couples $(\lambda, \gamma)$ for which percolation occurs:

- no percolation occurs when $\lambda<\lambda_{\mathrm{c}}$,

- no percolation occurs when $\gamma>1 / T$, 


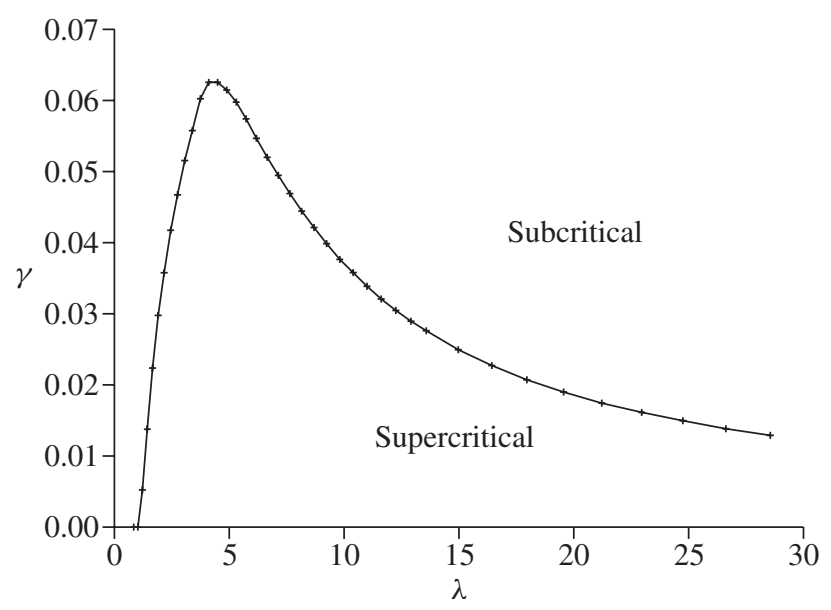

FIGURE 3: The percolation domain for $l(x)=\min \left(1, x^{-3}\right)$, computed by simulation.

- when $\lambda>\lambda_{\mathrm{c}}$, there exists a $\gamma^{*}(\lambda)>0$ such that percolation occurs whenever $\gamma<\gamma^{*}(\lambda)$,

- there exist $c_{1}<\infty$ and $\lambda^{\prime}<\infty$ such that $\gamma^{*}(\lambda) \leq c_{1} / \lambda$ for all $\lambda>\lambda^{\prime}$.

The last property follows from [2]. Figure 3 shows a simulation of the percolation domain for an attenuation function of the form $l(x)=\min \left(1, x^{-3}\right)$.

Finally, we mention a few possible extensions of our results. Firstly, our proof is twodimensional, at least the argument in Section 3.3 is. It is, therefore, a natural question as to whether our results can be extended to higher dimensions. From the application point of view, there is perhaps not so much interest in this question, but the mathematical challenges could be quite interesting. A second possible extension, which has more practical relevance, is to weaken the assumption of fixed power $P$. Instead of a fixed power $P$, we could argue that it is more realistic to have random, independent, and identically distributed transmitting powers for the points of the Poisson process. It would be interesting to see how moment conditions on $P$ would play their role in the analysis. Finally, there is the challenge to verify rigorously more properties of the curve in Figure 3.

\section{Acknowledgements}

The work presented in this paper was supported (in part) by the National Competence Center in Research on Mobile Information and Communication Systems (NCCR-MICS), a center supported by the Swiss National Science Foundation under grant number 5005-67322. Massimo Franceschetti was supported by the NSF Career grant CNS-0546235.

\section{References}

[1] Daley, D. J. (1971). The definition of a multidimensional generalization of shot noise. J. Appl. Prob. 8, 128-135.

[2] Dousse, O., Baccelli, F. and Thiran, P. (2005). Impact of interferences on connectivity in ad-hoc networks. IEEE/ACM Trans. Networking 13, 425-436.

[3] Gilbert, E. N. (1961). Random plane networks. J. SIAM 9, 533-543.

[4] Grimmett, G. (1999). Percolation, 2nd edn. Springer, Berlin.

[5] Meester, R. and Roy, R. (1996). Continuum Percolation. Cambridge University Press.

[6] Kingman, J. F. C. (1993). Poisson Processes (Oxford Stud. Prob. 3). Clarendon Press, Oxford. 